\title{
Procedimiento para caracterizar analizadores de descargas parciales de HF, VHF y UHF utilizados para el diagóstico de las redes e instalaciones de alta tensión
}

\section{Procedure for characterization of HF, VHF and UHF analisers used in high voltage installations diagnosis}

Fernando Garnacho ${ }^{1}$, Abderrahim Khamlichi ${ }^{2}$, Jorge Rovira ${ }^{3}$, Pascual Simón ${ }^{4}$, Ángel Ramírez ${ }^{5}$, Fernando Álvarez ${ }^{6}$

Garnacho, F; Khamlichi, A; Rovira, J; Simón, P; Ramírez, A; Álvarez, F. Procedimiento para caracterizar analizadores de descargas parciales de HF, VHF y UHF utilizados para el diagóstico de las redes e instalaciones de alta tensión. Tecnología en Marcha. Vol. 34, especial. ALTAE. Diciembre 2021. Pág 17-33.

doi) https://doi.org/10.18845/tm.v34i7.6009

LCOE-FFII. Universidad Politécnica de Madrid, España. Correo electrónico: fernando.garnacho@ffii.es

2 LCOE-FFII. Universidad Politécnica de Madrid, España. Correo electrónico: ak@ffii.es 


\title{
Palabras clave
}

Medidas de DP; Sensibilidad de DP; sensor de tipo HFCT; sensores de tipo VHF y UHF; ruido eléctrico; reconocimiento automático de defectos; Inteligencia artificial.

\section{Resumen}

La monitorización y medida de las descargas parciales en las redes eléctricas e instalaciones de alta tensión para evaluar el estado del aislamiento de los activos es un tema de gran actualidad, sin embargo, no existen criterios ni procedimientos para evaluar las características técnicas de estos sistemas de medida y diagnóstico. Los sistemas de cable se monitorizan con sensores de tipo HFCT por tener alcances de medida de varios kilómetros cada sensor. En las instalaciones GIS, los sensores invasivos de tipo UHF son ampliamente utilizados, al presentar alta sensibilidad para detectar pequeñas DP pese a su corto su alcance de pocos metros y para los transformadores de potencia se utilizan las capacidades de las bornas capacitivas, como condensador de acoplamiento acoplado a una impedancia de medida. Aunque aparentemente no existe una solución universal para resolver el mismo problema "detección del defecto del aislamiento eléctrico", en realidad no son bien conocidas las capacidades que tienen las diferentes técnicas de medida convencionales, en HF, VHF y las de UHF.

En este artículo se presenta un procedimiento de ensayo desarrollado con motivo del proyecto europeo "FuturEnergy" dentro del programa EURAMET que utiliza un banco de ensayo monofásico de alta tensión (20 kV) compuesto por una GIS y un sistema de cable que permite realizar pruebas de caracterización de sistemas de medida que utilicen sensores de tipo convencional, HFCT, VHF y de UHF. Las descargas parciales son generadas por un conjunto de probetas de referencia diseñadas al efecto o por un generador de trenes de pulsos de DP de referencia utilizado como calibrador.

\section{Keywords}

PD measurements; PD sensitivity; HFCT type sensor; VHF and UHF sensors; electrical noise; automatic recognition of defects; artificial intelligence.

\begin{abstract}
PD monitoring in electrical grids and high voltage installations to evaluate the insulation condition of different assets is a very topical issue, however, there are no criteria or procedures to evaluate the technical performances of these measuring and diagnosis systems. Cable systems are monitored with HFCT type sensors as each sensor has a measurement range of several kilometres. In GIS installations, invasive sensors of the UHF type are widely used, as they present high sensitivity to detect small PD despite their measuring range is a few meters and for power transformers the capacitance of its bushing is used, as a coupling capacitor coupled to a measuring impedance. Although there is apparently no universal solution to solve the same problem "detection of the electrical insulation defect", the capabilities of the different measuring techniques conventional, HF, VHF and UHF techniques are not well known.

This paper presents a test procedure using a single-phase high-voltage test bench (20 kV) composed of a GIS and a cable system that allows conducting tests of characterization of measuring systems working with conventional, HFCT, VHF or UHF sensors. Partial discharges are generated by a set of specially designed reference test cells or by a reference generator of PD pulses used as a calibrator.
\end{abstract}




\section{Introducción}

Las medidas de descargas parciales en las redes y en las subestaciones eléctricas forman parte no solo de los ensayos de recepción, sino también de las pruebas de mantenimiento de la gestión de activos. En los ensayos de recepción de subestaciones blindadas aisladas en gas (GIS) se utilizan generadores móviles de alta tensión alterna de tipo generador resonante ACR [1], los cuales son también utilizados para sistemas de cables de alta tensión de corriente alterna e incluso para cables de alta tensión continua (figura 1), mientras que en los sistemas de cables de media tensión se utilizan más frecuentemente generadores móviles de muy baja frecuencia VLF y de onda amortiguada DAC [1]. En todos ellos la medida de descargas parciales es comúnmente requerida a una tensión ensayo, por encima de la tensión de servicio (entre $\sqrt{3}$ y 3 dependiendo del tipo de generador utilizado y de la tensión nominal de la red) [2], [3] y [4]. En los ensayos de recepción se establece, como criterio de aceptación, la inexistencia de actividad de DP por encima de un umbral establecido (entre 5 pC y 10 pC dependiendo del tipo de instalación GIS o sistema de cable). Por este motivo se trata de realizar una medida a frecuencias normalizada (<1 MHz) conforme a los criterios de la norma IEC 60270 [5], aunque las interferencias características de las instalaciones de AT exigen, en ocasiones, realizar las medidas de DP en rangos de alta frecuencia $\mathrm{HF}(\leq 30 \mathrm{MHz})$, muy alta frecuencia VHF (entre 30 y $300 \mathrm{MHz}$ ) o incluso ultra alta frecuencia (UHF) (entre $300 \mathrm{MHz}$ y $3 \mathrm{GHz}$ ).

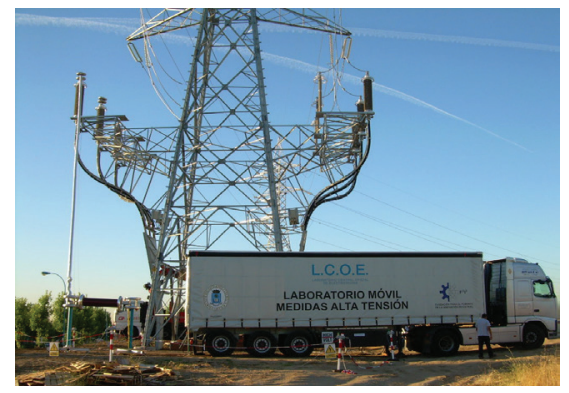

Figura 1. Generador resonante para ensayo de cable aislado de $220 \mathrm{kV}$.

Paralelamente, en los últimos años han proliferado las medidas de descargas parciales on-line en condiciones de servicio [6] muy útiles para el mantenimiento y gestión de activos. Medidas puntuales realizadas en periodos de tiempo relativamente cortos de unos pocos minutos permite identificar la presencia de actividad de descargas parciales en las redes o instalaciones. Sin embargo, la experiencia demuestra que en las redes de alta tensión (>100 kV), donde los equipos e instalaciones trabajan con altos niveles de campo eléctrico (> $9 \mathrm{kV} / \mathrm{mm}$ ) el fenómeno de la descarga disruptiva se acelera cuando aparecen los primeros síntomas de descargas parciales y consecuente el fallo eléctrico se desencadena en un menor periodo de tiempo en comparación con los defectos generadores de DP en los sistemas de media tensión, donde los gradientes de tensión no superan unos pocos kV/mm. En las redes de alta tensión (>100 $\mathrm{kV}$ ) no es eficiente plantearse un mantenimiento predictivo a través de medidas de descargas parciales periódicas durante cortos intervalos de tiempo realizadas cada varios años, por ejemplo 15 minutos cada tres años. Los sistemas de monitorización de descargas parciales permanentes son para las redes de alta tensión más eficientes, ya que permiten detectar de señales de descargas parciales justo cuando aparecen, y de esta forma poder actuar en los primeros síntomas de actividad de DP. En este sentido recientes investigaciones [7] han demostrado mayor capacidad de anticipación en la detección precoz de defectos midiendo las descargas parciales en los instantes justo después de una sobretensión transitoria de la red, por ejemplo, las producidas por maniobras o por descargas atmosféricas. La medida de las DP en los segundos posteriores a una sobretensión transitoria propia de la red permite la detección 
de señales de descargas parciales procedentes de defectos ocultos a las tensiones de servicio. Esta alternativa de medida resulta muy útil para detectar defectos de aislamiento que pudieran derivar en un fallo catastrófico cuando la actividad de descargas parciales se mantenga de forma continuada provocando un proceso acelerado que conduce a un fallo eléctrico en un corto intervalo de tiempo desde el inicio de la actividad de DP. Todos estos sistemas de medida de descargas parciales, tanto los utilizados en la recepción de las instalaciones eléctricas mediante generadores móviles, como los utilizados en las medidas de mantenimiento (medida temporal en cortos periodos de tiempo, monitorización continua o detectores de ráfagas de DP en los transitorios de la red) requieren métodos y criterios de verificación específicos aplicables a cada tecnología. Este artículo pretende contribuir en la futura normalización que debe desarrollarse a fin de cubrir la laguna normativa de estos sistemas de medida de DP y diagnóstico del aislamiento eléctrico.

\section{Campo de aplicación. Sistemas de media de DP que pueden caracterizar}

El campo de aplicación del procedimiento descrito en este artículo corresponde a aquellos sistemas de medida de DP que están constituidos por sensores electromagnéticos de alta frecuencia de tipo HFCT, VHF y UHF [8], uno o varios medidores (instrumentos de medida) de DP y un analizador de medidas de DP. Se entiende como analizador de medidas de DP cualquier elemento de hardware o software que realice un procesamiento de las señales de DP medidas a través de herramientas (por ejemplo, de inteligencia artificial) con el propósito de realizar un diagnóstico del estado del aislamiento eléctrico de la red o de la instalación de alta tensión.

\section{Probetas de referencia para la generación de descargas parciales}

Para el desarrollo de un banco de pruebas de caracterización de los medidores de DP utilizados en las medidas en campo es preciso reproducir de una forma fiel y controlada los defectos de aislamiento más representativos que pueden aparecer en las redes e instalaciones eléctricas. De esta forma se podrá determinar la sensibilidad de los instrumentos de medida en las condiciones reproducibles frente a defectos representativos. En el interior de cada una de las probetas se debe reproducir un solo defecto tipo. Los retos más difíciles de lograr en el diseño y construcción de las probetas es reproducir el defecto real de forma controlada que genere actividad de DP estable en un mismo rango de tensión para todas las probetas, en nuestro caso entre $18 \mathrm{kV}$ y $22 \mathrm{kV}$, a fin de reproducir los patrones resueltos en fase de los diferentes tipos de defectos a la misma tensión de ensayo sin que ésta sea un dato diferenciador de la probeta en ensayo.

Las probetas son caracterizadas [9], para conocer la tensión de inicio de DPs, y el nivel de DP a diferentes tensiones próximas a la tensión de ensayo, así como el tiempo máximo de uso. A pesar de ello es sabido que la estabilidad de las DP depende del tipo de defecto y las condiciones ambientales.

Las probetas-GIS tienen un aspecto diferente a las probetas-Cable-AIS, pero para cada una ellas dentro de estos dos grupos presentan el mismo aspecto externo, con una envolvente opaca, de modo que no se pueda identificar a simple vista, el tipo de defecto que encerrado en su interior. Únicamente el evaluador puede identificar el defecto en el interior de la probeta a través de un código, cuya correlación con el tipo de defecto sólo es conocida por el evaluador durante las pruebas de caracterización descritas en la siguiente sección. 
Probetas Cable-AIS relacionadas con defectos asociados a sistemas de cables aislado (Cable) de las redes eléctricas y sistemas aislados en aire (AIS)

Es importante clasificar diferentes tipos de defecto. En la figura 2 se muestran cuatro tipos de defectos de aislamiento asociados a redes eléctricas aisladas (cables) y sistemas aislados en aire (AIS). El defecto de corona en parte activa se puede generar en la práctica por elementos metálicos en punta de una conexión eléctrica en alta tensión. Los pulsos del efecto corona aparecen en, al menos, una de las crestas de la tensión, cuando es incipiente en la cresta de la polaridad negativa si la punta generadora de las DP corresponde con en la parte activa de alta tensión o en la cresta de polaridad positiva si la punta está la referida a tierra. El defecto de tipo superficial se produce en superficies contaminadas o degradadas, genera descargas parciales longitudinales que recorren la superficie del aislamiento. El patrón resuelto en fase de este defecto tiene como características comunes: 1) la asimetría de la aparición de DP en ambos semiperiodos, 2) los pulsos de DP aparecen únicamente en los cuartos de periodo en los que la tensión crece en valor absoluto, 3) en, al menos, un semiperiodo la densidad de las descargas se concentra formando un arco de luna que coincide con un crecimiento de la tensión en valor absoluto antes de la cresta de la tensión. En lo que respecta al defecto de cavidad en aislamiento sólido aparece por una burbuja de aire o gas al confeccionar el aislamiento eléctrico y se caracteriza por ser muy simétrico en ambos semiperiodos formando dos manchas cuando la tensión crece en valor absoluto y desaparece después de la cresta de la tensión. Los pulsos de DP pueden anticiparse al paso por cero de la tensión cuando la actividad es elevada. Por último, el defecto de potencial flotante se produce por herrajes metálicos no referidos a potencia fijo (tierra o alta tensión), normalmente no suele generar problemas críticos, pero en el caso de producirse en el interior de un recinto cerrado, como por ejemplo una cabina de MT, donde la ionización del aire puede concentrase excesivamente los efectos pueden ser críticos. Tal es el caso de la pérdida o falta de conexión a tierra de la pantalla de la terminación de un cable en el interior de una cabina de MT. El patrón resuelto en fase de este defecto es una nube de puntos de amplitud bastante constante que aparecer a uno y otro lado de los pasos por cero de la tensión.

\begin{tabular}{|l|l|l|}
\hline $\begin{array}{l}\text { Corona en parte } \\
\text { Activa }\end{array}$ & & \\
\hline $\begin{array}{l}\text { Descarga superficial } \\
\text { sobre aislante }\end{array}$ & & \\
\hline Cavidad & & \\
\hline Potencial Flotante & & \\
\hline
\end{tabular}

Figura 2. Tipos de defectos de aislamiento de las redes eléctricas aisladas y sus patrones resueltos en fase: a) corona en AT, b) partícula metálica en aislante, c) cavidad d) potencial flotante.

En la primera fila de la figura 3 se muestran los patrones de las probetas desarrolladas que permiten obtener los patrones resueltos en fase compatibles con los defectos reales y en la última fila de esta figura se muestran las probetas de dimensiones aproximadas de $6 \mathrm{~cm}$ de diámetro y $10 \mathrm{~cm}$ de altura, en cuyo interior se dispone el defecto. 


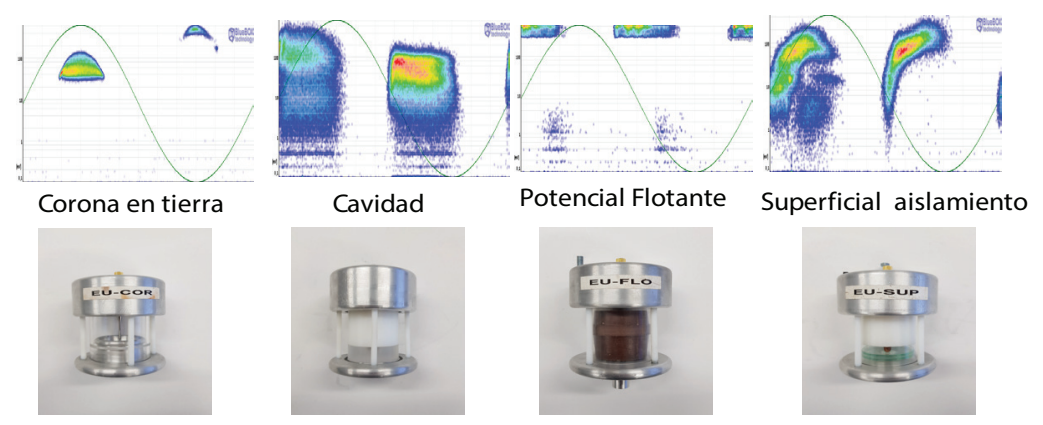

Figura 3. Fila superior: Patrones resueltos en fase de las probetas representativas de defectos: a) Corona en tierra, b) cavidad en contacto con AT, c) potencial flotante y d) superficial en aire.

\section{Probetas GIS relacionadas con defectos en GIS}

Los defectos tipo representativos de GIS se pueden clasificar en los cinco siguientes: partículas móviles, partículas sobre aislamiento, protusion, cavidad y potencial flotante. En la figura 4 se muestran los defectos de aislamiento tipo asociados a las subestaciones blindadas aisladas en gas (GIS). En la primera fila de la figura 5 se muestran los patrones resueltos en fase de defectos reales representativos en las GIS y en la segunda fila de la misma figura los patrones resueltos en fase obtenidos con las probetas que simulan los defectos.

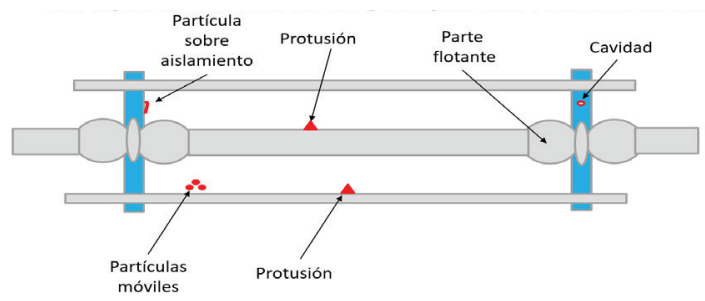

Figura 4. Tipos de defectos de aislamiento asociados a las GIS: a) partículas móviles, b) partículas sobre aislamiento, c) protusion, d) cavidad y e) potencial flotante.

Los patrones no son exactamente siempre iguales, sino que evolucionan en el tiempo de forma que guardan una serie de características comunes conocidas por los técnicos expertos o por los sistemas de inteligencia artificial, capaces de identificar el tipo de defecto. Es fundamental disponer de una amplia base de datos de los defectos tipo indicados con su evolución en el tiempo.

\begin{tabular}{|c|c|c|c|c|c|}
\hline $\begin{array}{l}\text { Tipo de } \\
\text { defecto }\end{array}$ & $\begin{array}{c}\text { Protusion } \\
\text { en } A T\end{array}$ & $\begin{array}{c}\text { Partículas } \\
\text { Móviles }\end{array}$ & $\begin{array}{l}\text { Potencial } \\
\text { flotante }\end{array}$ & $\begin{array}{l}\text { Partículas en } \\
\text { aislamiento }\end{array}$ & $\begin{array}{l}\text { Cavidad en } \\
\text { solido }\end{array}$ \\
\hline \multicolumn{6}{|c|}{$\begin{array}{l}\text { Defectos } \\
\text { reales }\end{array}$} \\
\hline \multicolumn{6}{|c|}{ Probetas } \\
\hline $\begin{array}{l}\text { Tipo de } \\
\text { defecto }\end{array}$ & $\begin{array}{l}\text { Protusion } \\
\text { en tierra }\end{array}$ & $\begin{array}{l}\text { Partículas } \\
\text { Móviles }\end{array}$ & $\begin{array}{l}\text { Potencial } \\
\text { flotante }\end{array}$ & Superficial & $\begin{array}{l}\text { Cavidad en } \\
\text { contacto con } \\
\text { electrodo }\end{array}$ \\
\hline
\end{tabular}

Figura 5. Fila superior: Patrones resueltos en fase de los tipos de defectos reales asociados a las GIS. Fila inferior: Patrones resueltos en fase de defectos tipo obtenidos en probetas-GIS. 


\section{Instalación de ensayos de caracterización de sistemas de medida de DP}

\section{Banco de ensayos}

El banco de ensayos consiste en una instalación GIL-Cable-AIS compuesta por un aislado en gas GIL de 0,5 m de diámetro y 6,0 m de longitud con cuatro compartimentos, dos de ellos con ventana accesible situados en los extremos (figuras 6 y 7). En cada una de estas ventanas se puede disponer un sensor invasivo de UHF (UHF1 y UHF2) o bien una probeta-GIS de DP relacionada con defectos en GIS para realizar las pruebas de caracterización. Al conducto GIL se conecta, por un lado, un cable de potencia de aproximadamente $50 \mathrm{~m}$ de longitud y por el otro lado, mediante una terminación-GIS, un conductor desnudo de unos $5 \mathrm{~m}$ de longitud que simula un sistema aislado en aire (AIS), al que se conecta el generador de alta tensión y el sistema de medida de DP de referencia compuesto por condensador de acoplamiento $C_{k} e$ impedancia de medida $Z_{m}$. El cable de potencia está compuesto por dos secciones de unos 25 m cada una, en cuya interconexión está preparada para colocar una probeta-Cable-AIS de DP. El banco de ensayo permite inyectar pulsos de DP por aplicación de alta tensión sobre las probetas o por conexión de generadores de señal: el generador de trenes de pulsos de DP de referencia de HF o el calibrador de UHF, descritos en las secciones 4.2 y 4.3. El banco de ensayos dispone de un generador de ruido eléctrico compuesto por un generador de funciones de ancho de banda de $30 \mathrm{MHz}$ y un transformador de corriente de alta frecuencia (HFCT) acoplado a la puesta a tierra de la pantalla del cable en la conexión con la GIL para inyectar el ruido eléctrico a las pantallas y la envolvente de la GIL. El sistema de medida de DP a caracterizar puede estar constituido por: (1) sensor de alta frecuencia HFCT y/o (2) sensor de UHF1, (3) medidor de DP y (4) analizador de medidas de DP. En determinados sistemas de medida se disponen también de convertidores UHF/HF (2)-bis para utilizar el mismo medidor de DP en alta frecuencia.

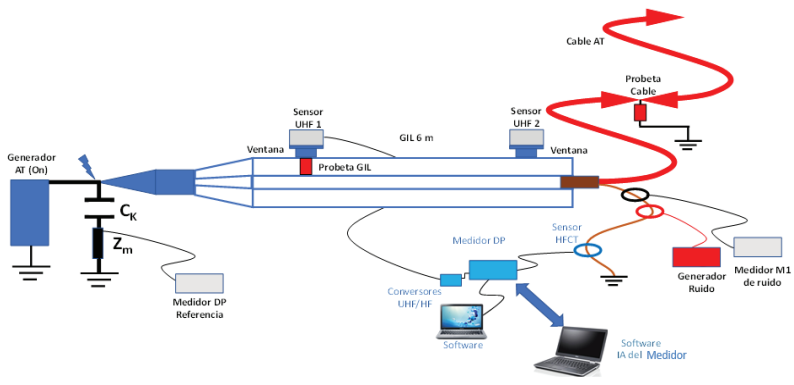

Figura 6. Esquema eléctrico del banco de ensayos desarrollado para caracterizar sistemas de medida compuestos por sensores, medidores con sus analizadores de DP.

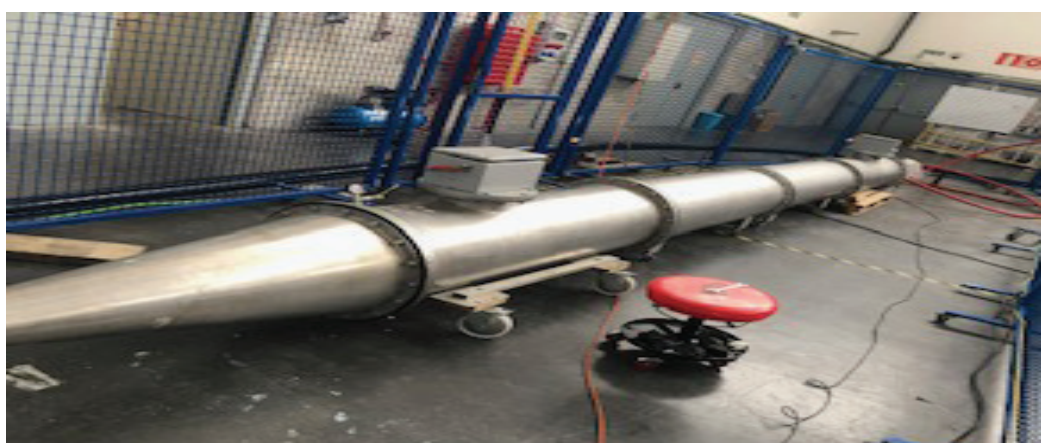

Figura 7. Vista externa del banco de ensayos de DP compuesto por GIL-cable-AIS. 


\section{Generador de trenes de DP de referencia en HF}

El generador de trenes de DP de referencia en HF es un generador de onda arbitraria de ancho de banda de, al menos $30 \mathrm{MHz}$, capaz de generar trenes de pulsos de DP de igual forma de onda con amplitudes individuales de los pulsos controlada $q_{1}, q_{2}, \ldots q_{n}$. El generador puede funcionar en modo "calibrador", a fin de generar trenes de pulsos de forma de onda $T_{1} / T_{2}$ definida y amplitud constante, $q_{0}$, en intervalos de tiempo equidistantes, inversamente proporcionales a la frecuencia de repetición de pulsos, N, elegida. El generador también puede funcionar en modo "generación de patrones de DP" (figura 8). A tal efecto, el generador cuenta con una amplia base de datos de trenes de DP asociados a cada uno de los defectos descritos en la sección 2, que fueron generados con las probetas de referencia o seleccionados por expertos cualificados de medidas realizadas en campo. Los trenes de pulsos de DP corresponden a defectos en Cable, subestaciones aisladas en aire o defectos procedentes de GIS detectados por sensores de tipo HFCT y UHF. Esta base de datos constituye la referencia experimental en la que se apoya la trazabilidad para evaluar la eficacia del reconocimiento de defectos tipo. Es deseable que otros institutos de investigación desarrollen otras bases de datos de estos mismos "defectos tipo" a fin de poder realizar comparaciones internacionales que conduzcan a la trazabilidad internacional de patrones de DP asociada a "defectos tipo generadores de DP". El proyecto europeo "FutureEnergy" soportado por EURAMET [10], actualmente en curso, en el que participan diferentes institutos metrológicos y universidades trabaja es esta dirección.

El generador dispone, además, la opción de generación de ruido eléctrico que puede superponerse a los trenes de pulsos generados en cualquier de los dos modos de trabajo. Se pueden generar dos tipos de ruido eléctrico: a) ruido blanco y b) ruido sinusoidal modulado de frecuencia fija, elegida por el generador de forma aleatoria en cada ensayo dentro de un rango de frecuencias preestablecido. El ruido blanco es el ruido más severo para cualquier medidor de DP, al no existir ninguna frecuencia específica en la que la señal/ruido sea máxima. El ruido sinusoidal modulado simula señales de emisoras de radiofrecuencia que es más sencillo de rechazar a través de selección de la banda de medida. A título de ejemplo este tipo de ruido puede ser rechazado utilizando el método de medida de banda estrecha acorde a la IEC 60270. Además del canal de salida de DP indicado en los párrafos anteriores, el generador dispone de un segundo canal de salida para generar la señal de tensión de alterna de frecuencia de red $(50 \mathrm{~Hz} \circ 60 \mathrm{~Hz})$ que permita construir el patrón resuelto en fase. En la figura 10 se muestra una vista del generador de trenes de pulsos de referencia generados en modo "calibrador" y en modo "generación de patrones".

\section{Calibrador en UHF}

Cuando se requiera inyectar señales que simulen los pulsos de descargas parciales producidos en el interior de la GIS que deban ser detectados por sensores de tipo UHF se utiliza un calibrador de UHF con pulsos de ancho $<2$ ns y tiempos de subida $<0,5$ ns.
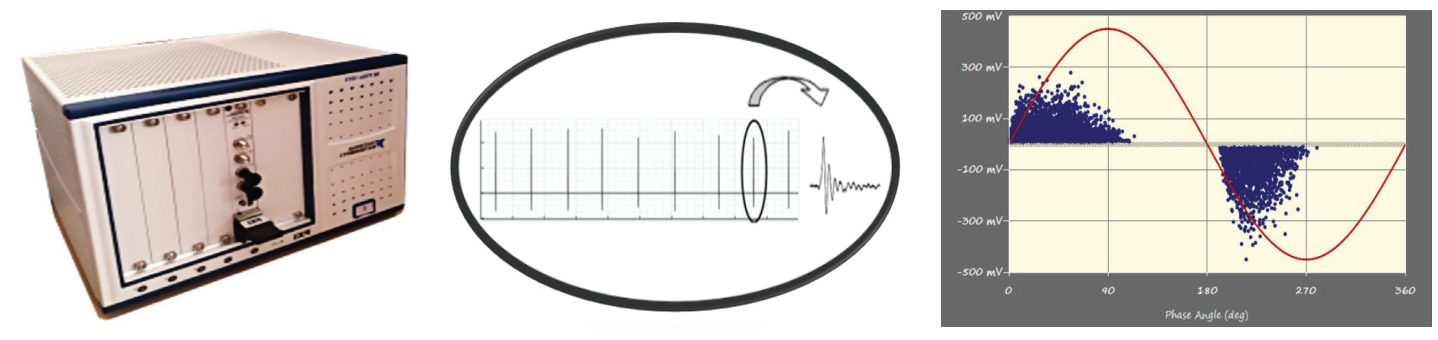

Figura 8. Generador de trenes de pulsos de DP de referencia de HF: a) vista externa, b) tren de pulsos en modo "calibrador", c) Patrón de tren de pulsos de defecto tipo cavidad. 


\section{Pruebas de caracterización de sistemas de medida de DP y diagnóstico}

Las pruebas de caracterización de los diferentes sistemas de medida de DP y diagnóstico del aislamiento utilizados en ensayos in situ de las redes e instalaciones eléctricas deben ser conformes a los criterios de la norma IEC 60270 o a la especificación técnica TS IEC 62478 en función de su aplicación. Las medidas de DP en los sistemas de cable de alta tensión y en las GIS se utilizan para evaluar el estado del aislamiento en los ensayos de recepción y en las verificaciones de mantenimiento a través de la monitorización continua. Los ensayos de recepción se realizan mediante generadores móviles (resonantes ACR, de muy baja frecuenciaVLF o de onda amortiguada DAC), mientras que las medidas de DP en las monitorizaciones continuas se realizan en condiciones de normales de servicio (medidas on-line) utilizando la propia red de potencia como fuente de alta tensión. Aunque en los ensayos de recepción es deseable realizar las medidas de DP a frecuencias por debajo de $1 \mathrm{MHz}$ conforme a criterios de la IEC 60270, no siempre es posible obtener la sensibilidad requerida en las condiciones de ruido de la instalación. Por ello, en muchas ocasiones es preciso efectuar las medidas a frecuencias por encima de $1 \mathrm{MHz}$ recurriendo a métodos conformes a la TS IEC 62478. Para los ensayos de recepción la magnitud de referencia a considerar es la carga aparente, expresada en pico-Culombios. Incluso si la frecuencia de medida es superior a $1 \mathrm{MHz}$, siempre que no supere algunos megahercios, es posible expresar la medida de DP en pico-Culombios cuando se realiza un ensayo con generador móvil. Sin embargo, en las monitorizaciones de DP conectados a la red de potencia no es posible realizar medidas de DP con trazabilidad en pC, , ya que para lograrlo sería preciso inyectar una señal de calibración justo en punto donde en defecto aparece, el cual es evidentemente desconocido a priori y en la mayoría de los casos sería inaccesible la inyección en tal punto. En estos casos, los parámetros de referencia a exigir a los analizadores de DP con dos: 1) sensibilidad mínima, expresada en pC, en condiciones normalizadas y 2) umbral de eficacia para identificar diferentes tipos de patrones representativos de defectos críticos, expresada en \%. En este último caso, se requieren patrones de DP resueltos en fase de referencia que permitan obtener trazabilidad requerida.

\section{Ensayos aplicables a los sensores de DP}

\section{Determinación de la impedancia de transferencia Z(f) del sensor}

La norma IEC 60270 define como impedancia de transferencia Z(f) la relación entre la amplitud de salida $V_{\text {out }}$ y la amplitud constante de la corriente de entrada, $I_{\text {in, }}$ en función de la frecuencia, cuando la corriente de entrada es sinusiodal. Debe destacarse que la impedancia de transferencia $Z(f)$ tiene una relación directa con la sensibilidad de la medida ya que cuanto mayor sea la señal de salida $V_{\text {out }}$ frente a un $1 \mathrm{~mA}$ de la corriente del pulso de entrada, el instrumento de medida podrá medirlo mejor. El ensayo requiere el uso de un generador sinusoidal de frecuencia variable a fin de determinar la función de transferencia tanto en amplitud como en fase del elemento utilizado como transductor de la señal de DP, cuando la salida del este elemento está conectado a una impedancia de $50 \Omega$ que representa el instrumento de medida. El elemento transductor podrá ser la impedancia de medida, $Z_{m}$, utilizada en serie con condensador de acoplamiento, $C_{k}$, (método conforme a la norma IEC 60270) o podrá ser un sensor de alta frecuencia HF, como por ejemplo un transformador de corriente HFCT, o un sensor de tipo antena de VHF o UHF. En cualquier caso, el elemento transductor utilizado transforma la señal de los pulsos de las DP en una señal de tensión adaptada al instrumento de medida (normalmente un registrador digital). 
Cuadro 1. Ensayos de los sistemas de medida de DP para el diagnóstico del aislamiento.

\begin{tabular}{|c|c|c|c|c|}
\hline Tipo de ensayo in situ & \multicolumn{2}{|c|}{$\begin{array}{c}\text { Ensayo de recepción } \\
\text { IEC } 60270 \\
\text { (TS IEC 62478) }\end{array}$} & \multicolumn{2}{|c|}{$\begin{array}{c}\text { Monitorización DP } \\
\text { TS IEC } 62478 \\
\text { (on line) }\end{array}$} \\
\hline Aplicación & Cable & GIS & Cable\&GIS & GIS \\
\hline Fuente de alta tensión utilizada & $\begin{array}{l}\text { Generador } \\
\text { Móvil } \\
\text { ACR }^{1}, V_{L} F^{2}, \\
\text { DAC }^{3}\end{array}$ & $\begin{array}{l}\text { Generador } \\
\text { Móvil ACR }^{1}\end{array}$ & \multicolumn{2}{|c|}{$\begin{array}{c}\text { Red de potencia de media } \\
\text { y alta tensión }\end{array}$} \\
\hline Rangos de frecuencia de medida DP & \multicolumn{2}{|c|}{$\begin{array}{c}\mathrm{f}_{\mathrm{m}} \leq 1 \mathrm{MHz} \\
\left(1 \mathrm{MHz}<\mathrm{f}_{\mathrm{m}} £ \quad 3 \mathrm{GHz}\right)\end{array}$} & $\begin{array}{r}H F \\
f_{m} \leq 30 M H z\end{array}$ & $\begin{array}{l}f_{m}>30 M H z \\
f_{m} \leq 3 \mathrm{GHz}\end{array}$ \\
\hline Parámetro de Referencia trazabilidad & \multicolumn{2}{|c|}{$\mathrm{q}_{\mathrm{IEC}}(\mathrm{pC})$} & \multicolumn{2}{|c|}{$\begin{array}{l}\text { Sensibilidad } q_{1 E C}(p C) \text { y } \\
\text { Patrón resuelto en fase }\end{array}$} \\
\hline Tipo de sensor & $\mathrm{Z}_{\mathrm{m}} / \mathrm{HFCT}$ & $\mathrm{Z}_{\mathrm{m}} /(\mathrm{UHF})$ & HFCT & VHF-UHF \\
\hline \multicolumn{5}{|l|}{ 5.1) Ensayos a los sensores de DP } \\
\hline 5.1.1) Impedancia de transferencia Z(f) & IEC 60270 & IEC 60270 & $>5 \mathrm{mV} / \mathrm{mA}$ & en estudio \\
\hline 5.1.2) Rango de frecuencia $f_{1}$ y $f_{2}$ de $Z$ (f) & $\leq 1 \mathrm{MHz}$ & $\leq 1 \mathrm{MHz}$ & $1-30 \mathrm{MHz}$ & $0,03-3 \mathrm{GHz}$ \\
\hline 5.1.2) Factor escala a la $f_{m}$ & $\pm 5 \%$ & $\pm 5 \%$ & $\begin{array}{l} \pm 10 \% \\
\left(f_{A}, f_{B}\right)\end{array}$ & $\begin{array}{l}\text { en estudio } \\
\qquad\left(f_{A}, f_{B}\right)\end{array}$ \\
\hline \multicolumn{5}{|l|}{ 5.2) Ensayos al medidor de DP } \\
\hline 5.2.1) Medida de carga aparente $q$ y $N$ & $\begin{array}{c}q_{\mathrm{IEC}}, N \leq \pm \\
2 \%\end{array}$ & $q_{\mathrm{IEC}}, N \leq \pm 2 \%$ & \multicolumn{2}{|c|}{$N \leq \pm 10 \%$} \\
\hline 5.2.2) Error de medida q, N vs ruido. & $q<10 \%$ & $q<10 \%$ & \multicolumn{2}{|c|}{$N \leq \pm 30 \%$} \\
\hline \multicolumn{5}{|l|}{ 5.3) Ensayos al sistema medida DP } \\
\hline 5.3.1) Sensibilidad & $<10 \mathrm{pC}$ & $<5 \mathrm{pC}$ & $<10 \mathrm{pC}$ & $<5 \mathrm{pC}$ \\
\hline 5.3.2) Eficacia para identificar defectos & Experto & Experto & $>80 \%$ & $>80 \%$ \\
\hline 5.3.3) Eficacia para separar defectos & Experto & Experto & ${ }^{3} 4$ defectos & ${ }^{3} 4$ defectos \\
\hline 5.3.4) Eficacia para localizar de defectos & $<5 \mathrm{~m}$ & $<30 \mathrm{~cm}$ & $<5 \mathrm{~m} / 30 \mathrm{~cm}$ & $<30 \mathrm{~cm}$ \\
\hline
\end{tabular}

(1)ACR: Resonante de CA (2)VLF: Muy baja frecuencia, ${ }^{(3)} \mathrm{DAC}$ : onda amortiguada de CA.

Para una sistema de medida conforme a la norma IEC 60270 que mida en banda estrecha (9 $\mathrm{kHz} \leq \Delta \mathrm{f} \leq 30 \mathrm{kHz}$ y $50 \mathrm{kHz} \leq \mathrm{fm} \leq 1 \mathrm{MHz})$ o en banda ancha $(30 \mathrm{kHz} \leq \mathrm{fl} \leq 100 \mathrm{kHz}, \mathrm{f} 2 \leq 500 \mathrm{kHz}$ y $100 \mathrm{kHz} \leq \Delta f \leq 400 \mathrm{kHz}$ ) es sabido que el valor de cresta del pulso a la salida de la impedancia de medida, $Z_{m}$, es proporcional al valor de la carga del pulso de corriente de entrada, lo cual permite establecer una trazabilidad directa entre la magnitud carga del pulso (pC) y la tensión medida (mV). Sin embargo, cuando se miden las DP por métodos no convencionales, diferentes a los descritos en la norma IEC 60270, que funcionan en rangos de frecuencias superiores a 1 $\mathrm{MHz}$, no es posible establecer la referida trazabilidad directa entre carga y tensión a la salida del sensor para cualquier forma de onda arbitraria de pulso de DP. En este caso, para establecer la trazabilidad de la medida con el valor de la carga del pulso es preciso, en primer lugar, asegurarse que el ancho de banda del sensor capte el contenido frecuencial representativo de los pulsos de DP. Ello permitirá establecer dos diferentes estrategias de medida. La más rigurosa, pero a la vez más difícil, consiste en realizar una integración efectiva del pulso, a partir de la medida de respuesta en frecuencia del sensor. El método consiste en reconstruir 
el pulso original a través de la característica de amplitud y fase en función de la frecuencia de la impedancia de transferencia del sensor. La otra estrategia menos rigurosa, pero mucho más sencilla, es establecer la relación entre la carga del pulso, $\mathrm{q}_{\mathrm{i}}$, y la señal de tensión medida para una determinada frecuencia de medida en la que el pulso contenga una amplitud significativa respecto de la señal de ruido. En este último caso, es preciso asegurarse que el contenido frecuencial de los pulsos a medir durante el ensayo con alta tensión sea el mismo que los pulsos utilizados en la calibración donde se establece la correlación entre pC y mV para la frecuencia de medida. Este es el principio en el que se basa el ensayo de sensibilidad referido en la especificación técnica TS IEC 62478 que requiere dos pasos para su correcta ejecución. El primer paso en laboratorio donde se establece la relación entre pC y mV para pulsos de DP producidos por un defecto real y los generados sintéticamente a través de calibrador de DP con contenido frecuencial análogo a los pulsos de DP del defecto real y el segundo paso en campo, dónde se chequea la sensibilidad en condiciones locales de ruido, mediante el generador sintético según se describe en 5.2.1.

En la figura 9 se muestra la respuesta en frecuencia de la impedancia de transferencia para tres variantes de un mismo sensor de tipo transformador de corriente HFCT: a) sensor sin apantallamiento con una sola bobina de medida de DP, b) sensor sin apantallamiento con una bobina de media y otra adicional de inyección de pulsos utilizada para chequeo del sensor y c) sensor igual al b) que incluye, además, un apantallamiento de cobre para mejorar su inmunidad frente al ruido eléctrico. Puede observarse que la respuesta en frecuencia de las tres alternativas de sensor es análoga para frecuencias inferiores a $10 \mathrm{MHz}$. Para frecuencias inferiores a 300 $\mathrm{kHz}$ la relación entre la tensión de salida del sensor y la corriente de entrada es proporcional a la frecuencia y por lo tanto el sensor se comporta como una impedancia inductiva de valor $\mathrm{U} / \mathrm{I}$, proporcional a la frecuencia. Para frecuencias por encima de $1 \mathrm{MHz}$ y hasta la frecuencia de corte superior $\mathrm{f}_{2}$ el sensor se comporta como un transformador de corriente cuya corriente secundaria viene dada por $V_{\text {out }} / 50 \mathrm{~W}$.
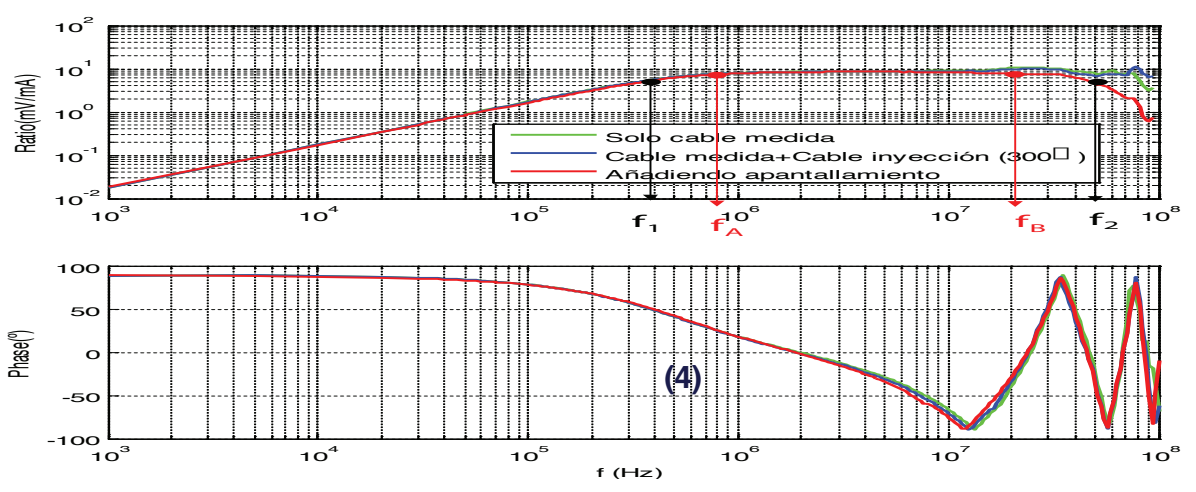

Figura 9. Respuesta en frecuencia de la relación y fase de tres variantes del mismo sensor HFCT: con una sola bobina de medida sin apantallamiento, con dos bobinas una de medida y otra de chequeo sin apantallamiento y c) igual que el b) y con apantallamiento.

Rango de frecuencias de corte $f_{1}$ y $f_{2}$ del sensor $y$ rango de frecuencias $f_{\mathrm{A}}$ y $f_{\mathrm{B}}$ donde el factor de escala permanece constante.

Aunque la norma IEC 60270 establece como frecuencias de corte inferior y superior, $f_{1}$ y $f_{2}$, de una impedancia de la medida, $Z_{m}$, cómo aquellas para las cuales la respuesta en frecuencia se reduce -6 dB con relación al valor máximo $\left(Z_{m}\right.$ cae al $50 \%$ del valor máximo), es preciso establecer un rango de frecuencias más estrecho de trabajo, $f_{A}$ y $f_{B}$ (figura 11), para lograr que el factor de escala del sistema de medida esté dentro del $\pm 5 \%$ (máxima variación de $Z_{m}=10 \%$ ). 
Téngase en cuenta que el factor de escala de un sistema de medida de DP conforme a la norma IEC 60270 viene definido principalmente por su impedancia de medida, $Z_{m}$. Para el caso de sensores de HF, VHF o UHF utilizados en medidas no convencionales de monitorización conformes a la TS IEC 62478 se establece como límite de frecuencias de trabajo $f_{A}$ y $f_{B}$ el doble que para un sistema de medida conforme a la IEC $60270( \pm 5 \%)$. En la figura 11 se muestran las frecuencias de corte inferior y superior, $f_{1}=380 \mathrm{kHz}$ y $\mathrm{f}_{2}=50 \mathrm{MHz}$ para un sensor de tipo HFCT apantallado, así como sus frecuencias útiles de trabajo, $\mathrm{f}_{A}=0,8 \mathrm{MHz}$ y $\mathrm{f}_{B}=20 \mathrm{MHz}$ para las cuales el factor de escala no varía más del $\pm 10 \%$. En la figura 10 se muestran las impedancias de transferencia $Z(f)$ de cinco sensores comerciales diferentes. Los sensores (1) y (2) presentan valores de impedancia de transferencia elevados (>8 $\mathrm{mV} / \mathrm{mA}$ ) en el rango de frecuencia entre de $0,5 \mathrm{MHz}$ a $20 \mathrm{MHz}$, pero no mantienen constante su valor, con una diferencia máxima de $Z(f)=-50 \%$ para el sensor (1) y del $900 \%$ para el sensor (2). Los sensores (3) y (4) aunque mantienen constante su respuesta en frecuencia en el rango de frecuencias referido (máxima variación de $Z(f)<10 \%)$ presentan bajos valores de impedancia de transferencia $(<5 \mathrm{mV} / \mathrm{mA})$ que afecta negativamente a la sensibilidad de la medida. El sensor (5) mantiene un compromiso entre valor de la impedancia de transferencia máximo $(8,3 \mathrm{mV} / \mathrm{mA})$ y la constancia de la misma $(<10 \%)$ en el rango de frecuencias de interés $(0,5 \mathrm{MHz}$ a $20 \mathrm{MHz})$.

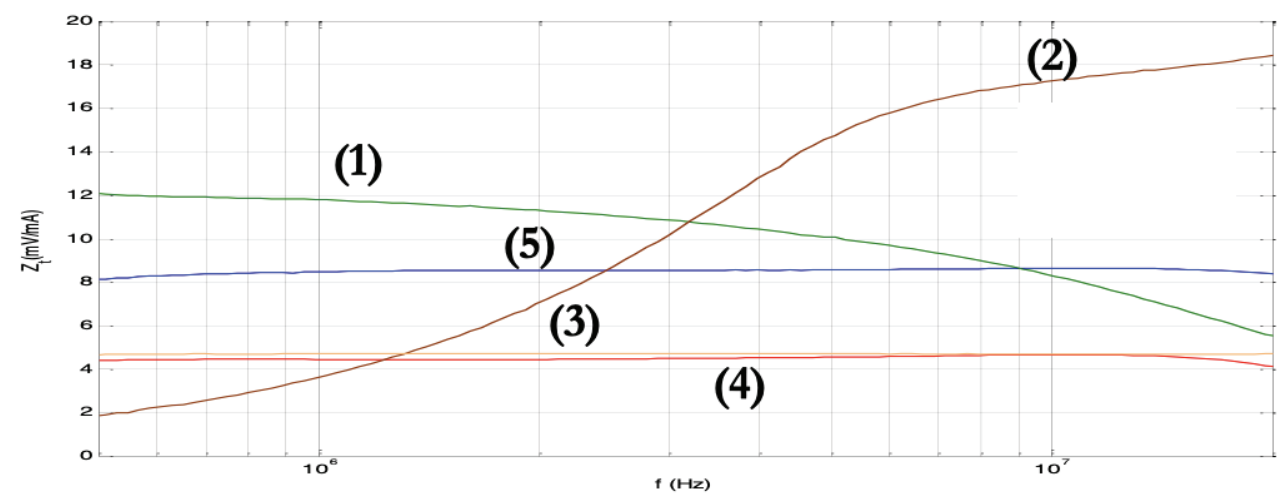

Figura 10. Respuesta en frecuencia de cinco sensores comerciales de tipo HFCT: (1) y (2) sensores de alto valor de Z(f) pero no constante.

\section{Ensayos aplicables a los medidores y analizadores de DP}

Las pruebas referidas en esta sección son solo aplicables a los medidores y software de análisis que incorporan los sistemas de medida para evaluar el estado del aislamiento.

\section{Medida de carga aparente qIEC y frecuencia de repetición de pulsos $N$.}

La carga aparente, $q_{\mathrm{IEC}}$, expresada habitualmente en picoculombios ( $\mathrm{pC}$ ), se define en la norma IEC 60270 como la carga que, si se inyecta en un tiempo muy corto entre los terminales del objeto de ensayo dispuesto en un circuito de ensayo especificado, daría la misma lectura en el instrumento de medida que el propio pulso de corriente de DP generado por un defecto en el seno del aislamiento. Además, la norma establece que para ensayos de corriente alterna, el valor especificado de la carga aparente $q_{\text {IEC }}$ es el valor de la mayor descarga parcial repetitiva. Para cuantificar el valor de la mayor descarga parcial repetitiva la norma IEC 60270 utiliza un circuito analógico de detección de cresta con una constante de tiempo de carga muy corta y una constante de tiempo de descarga no mayor de 0,44 s. La norma establece la máxima tolerancia aceptable de la lectura de un instrumento de medida de DP frente a trenes de pulsos equidistantes y de igual valor de carga entre ellos. La frecuencia de repetición de 
pulsos, N, debe variar entre 1 pulso/s hasta valores superiores a 100 pulsos/s. En la figura 11 se muestran los límites establecidos por la norma para verificar la lectura de los medidores de carga aparente. El generador de trenes de pulsos de DP de referencia descrito en la sección 4.2 permite generar pulsos de igual amplitud equidistantes en intervalos de tiempo controlables desde 1 pulso/s hasta 100 pulsos/s (véase parte derecha de la figura 11) para verificar que la lectura del medidor se encuentra en el rango de tolerancia referido en la figura.

\begin{tabular}{|c|c|c|c|c|c|c|}
\hline $\mathrm{N}(1 / \mathrm{s}):$ & 1 & 2 & 5 & 10 & 50 & $\geq 100$ \\
\hline $\mathrm{R} \min (\%):$ & 35 & 55 & 76 & 85 & 94 & 95 \\
\hline Rcalc (\%): & 36.7 & 57.1 & 78.7 & 88.5 & 97.5 & 98.76 \\
\hline$R_{\max }(\%):$ & 45 & 65 & 86 & 95 & 104 & 105 \\
\hline
\end{tabular}

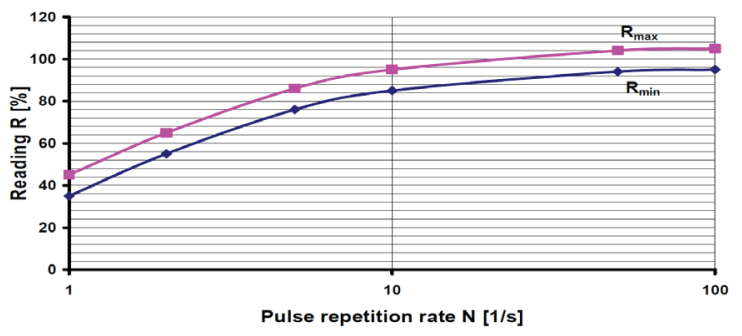

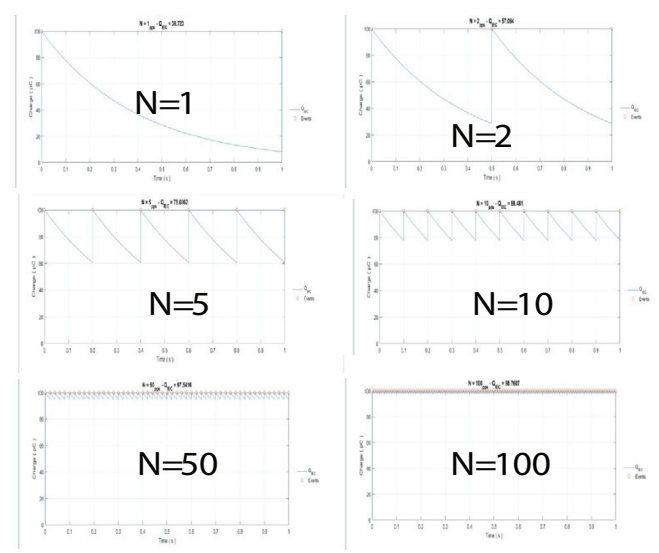

Figura 11. Tolerancia máxima admisible de la lectura del instrumento de medida en función de la frecuencia de repetición de los pulsos de DP, N.

Cuando se incrementa la frecuencia de repetición por encima hasta 1 pulso/10us es posible determinar el tiempo de resolución de pulsos $T_{r}$, del medidor de DP. El tiempo de resolución es definido por la norma como el intervalo de tiempo más corto entre dos pulsos consecutivos para los cuales el valor de cresta medido no cambia más del 10\% respecto al valor de carga cuando se mide uno solo pulso y representa la capacidad para diferenciar eventos de DP sucesivos.

\section{Error de medida DP vs ruido}

El generador de trenes de pulsos de DP de referencia en modo "calibrador" se conecta al medidor de DP, para generar trenes de pulsos de forma de onda $T_{1} / T_{2}$ y amplitud, $q_{0}$, constante, secuenciados en intervalos de tiempo equidistantes. Simultáneamente, los pulsos de DP se mezclan con ruido eléctrico, primero con ruido blanco y luego con ruido modulado a fin de determinar la sensibilidad para detectar pulsos sin ruido eléctrico y en las dos condiciones de diferente tipo de ruido.

El primer paso consiste en seleccionar la frecuencia central de medida y el ancho de banda de medida del medidor que permita mitigar, lo mejor posible el ruido eléctrico. A tal fin el generador generará el mismo tipo de ruido que se utilizará durante la prueba, que es mezclado con un tren de pulsos de DP de amplitud suficientemente grande como para poder ser reconocido fácilmente (por ejemplo 200 pC de señal de DP frente a un ruido de 50 pC). Se realizarán los ajustes necesarios en el medidor para lograr la mejor relación señal/ruido, si el equipo dispone de capacidad de rechazo de ruido automático no será necesario efectuar ajuste alguno, de lo contrario se seleccionará el nivel de disparo, la frecuencia central de medida y ancho de banda del medidor más apropiados dentro de los rangos de frecuencia requeridos para la prueba (ver cuadro 1). Estos ajustes no deben ser cambiados durante el resto del ensayo para el tipo de ruido en el que se determinará la sensibilidad vs ruido. Tras el ajuste indicado se procede a seleccionar la ganancia del medidor para que la lectura del medidor corresponda a pulsos de calibración de 200 pC. 
El ensayo consiste en realizar la medida del valor amplitud de las DP (expresado en pC, mV o $\mathrm{dBm}$ ) y la frecuencia de repetición, $\mathrm{N}$, que el generador inyecta al medidor para cuatro niveles carga aparente $\mathrm{q}_{\mathrm{IEC}}$ y cuatro niveles del ruido eléctrico (ruido muy alto, ruido alto, ruido medio y sin ruido). En cada uno de los cuatro valores de carga $\mathrm{q}_{\mathrm{IEC}} \mathrm{X}_{1}, \mathrm{X}_{2}, \mathrm{X}_{3}$ y $\mathrm{X}_{4}$ el generador variará el nivel de ruido eléctrico inyectado de forma decreciente, desde el nivel más alto de ruido hasta sin ruido. Los cambios se realizan por el generador tras la introducción de las lecturas de amplitud de la DP y la frecuencia de repetición, N, efectuadas por el medidor a caracterizar correspondientes al tren de pulsos generado. Los valores de carga inyectada se varían entre un valor mínimo $\mathrm{q}_{\min }(5 \mathrm{pC}$ o $10 \mathrm{pC})$, y uno máximo $\mathrm{q}_{\max }(200 \mathrm{pC})$ en dos intervalos de 50 pC y 100 pC. Consecuentemente, se realizarán 16 pares de lecturas (amplitud de la DP y frecuencia de repetición N) que permiten representar curvas error de medida vs frecuencia como las mostradas en la figura 12 para un determinado medidor bajo caracterización y frente a los dos tipos de ruido: sinusoidal modulado y ruido blanco respectivamente. Se evidencia la gran diferencia de errores del medidor en función del tipo de ruido eléctrico considerado modulado o blanco.
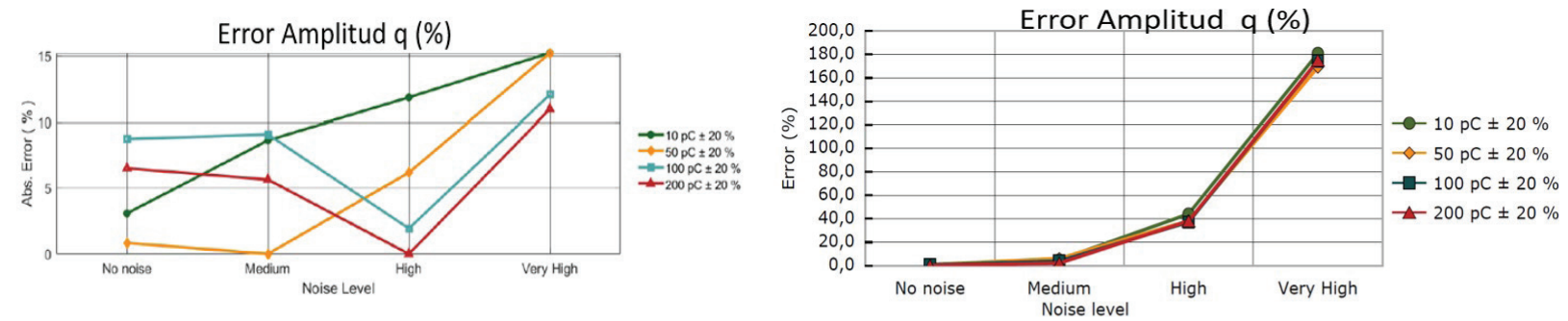

Error N (\%)
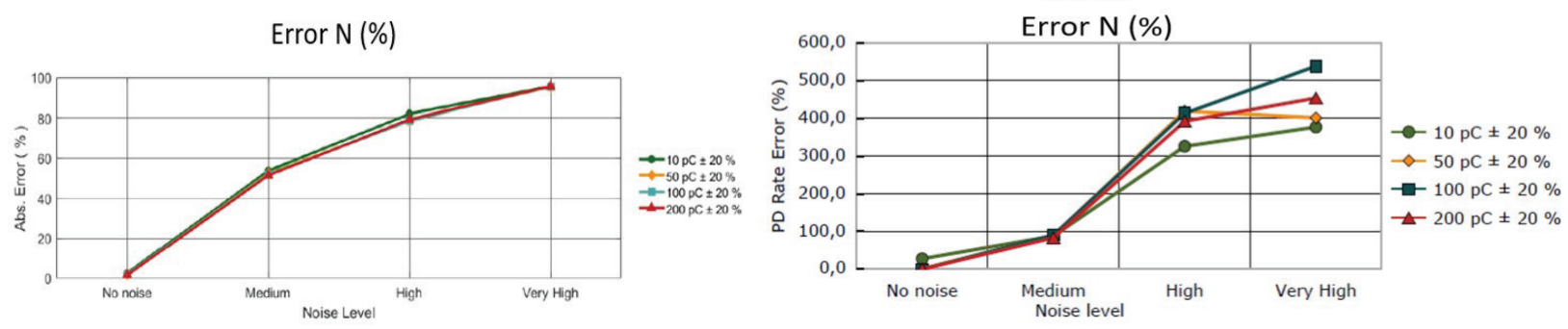

Figura 12. Familia de curvas del ensayo sensibilidad vs ruido: a) ruido modulado, b) ruido blanco.

\section{Ensayos aplicables al sistema de medida de PD y analizador en conjunto}

\section{Ensayos de sensibilidad}

Los ensayos de sensibilidad del sistema de medida consisten en inyectar señales de DP en el banco de ensayo descrito en 4.1, primero producidas con probetas de referencia y luego con el generador de pulsos o calibrador de DP a fin de identificar la mejor sensibilidad entre 1 pC y 10 pC.

Para establecer la sensibilidad del sistema de media a caracterizar cuando se mide con sensores de tipo UHF o con sensores de tipo HFCT cuando aparecen defectos en la GIS se sigue el método de dos pasos referido en la "TS IEC 62478".

Paso 1: Tras realizar la calibración del sistema de medida de referencia de DP, se dispone de la probeta-GIL de tipo partículas móviles en la ventana del sensor UHF1 y se aplica alta tensión hasta la aparición estable de actividad de DP. Seguidamente se realiza la medida sincronizada de los pulsos de descargas parciales generados por la probeta, a través del o de los sensores 
del sistema de medida a caracterizar, y a través del sistema de medida de DP de referencia a fin de establecer la relación entre la magnitud de salida del medidor y la medida de referencia expresada en pC.

Paso 2: Tras desconectar la alta tensión y extraer la probeta en la GIL, se inyecta señales de UHF generadas con un calibrador de UHF si se desea caracterizar un medidor de UHF o señales de HF generadas a través del generador de trenes de DP de referencia en HF, si se desea caracterizar un medidor de HF, según proceda (figura 9). La inyección de los pulsos se realiza a través de la ventana del sensor UHF2 y la amplitud de los pulsos será inicialmente lo suficientemente grande para que el sensor UHF1 o el sensor de HFCT bajo caracterización detecten los pulsos generados. Seguidamente, se reducen la amplitud de los pulsos inyectados hasta que el sistema de medida bajo caracterización no pueda distinguirlos de otros del ruido.

La sensibilidad frente a defectos en cable se determina solo cuando el sistema de medida utiliza sensores de tipo HFCT. A tal fin se repite el ensayo descrito en el apartado anterior con la diferencia de que la probeta elegida es la de tipo cavidad situada en la interconexión de las dos secciones de cable y que la inyección de las señales de DP se efectúa con el generador de trenes de DP de referencia en HF situado en la interconexión de las dos secciones de cable (figura 11) y se mide solo con el sensor de tipo HFCT.

\section{Eficacia para la identificación automática de defectos tipo}

La eficacia para identificar automáticamente el tipo de defecto se realiza mediante una prueba en alta tensión mediante las nueve probetas de referencia descritas en la sección 3, todas ellas con envolvente opacas desde el exterior, a fin de no poder ver el defecto encerrado en su interior. Adicionalmente, se utilizan dos probetas "no activas" de DP, es decir sin defecto en su interior, una probeta Cable-GIS y otra Probeta-GIS. El evaluador debe seleccionar una probeta con defecto de entre las nueve disponibles para colocarla en uno de los dos emplazamientos previstos al efecto: en la GIL o en el cable, y otra "no activa" para colocarla en el otro emplazamiento disponible. Sólo el evaluador debe conocer el tipo de defecto seleccionado en cada caso a resolver. Tras la colocación de las dos probetas, una con defecto y otra sin él, se incrementa la tensión del generador que alimenta al banco de ensayos de forma creciente hasta alcanzar la tensión de ensayo de $20 \mathrm{kV}$. Se realiza la medida de descargas parciales a través del sistema de DP de referencia, por el método normativo IEC 60270, con objeto de asegurar la actividad de DP a la tensión de ensayo. Seguidamente se inyecta, a través del generador de ruido eléctrico del banco de ensayos, un ruido eléctrico de nivel alto para asegurar que el patrón queda oculto en el sistema de medida de referencia. La amplitud del ruido eléctrico debe ser un $20 \%$ por encima de la amplitud de los pulsos de DP expresándolo en valor de ruido eléctrico en pC y medido por el sistema de medida de DP de referencia. La tensión de ensayo se mantiene para que el sistema de medida a caracterizar capture las medidas oportunas y realice el procesamiento de la señal que precise a fin de obtener el resultado del tipo de defecto identificado. Solo resultados con un nivel de confianza superior al $80 \%$ declarados por el sistema de inteligencia artificial del sistema de medida a caracterizar serán considerados. Seguidamente se repite la prueba para el nivel bajo de ruido eléctrico correspondiente al 25\% de la amplitud de las DP.

\section{Eficiencia en la separación de fuentes de defectos tipo}

La evaluación de la eficacia para separar los defectos automáticamente se realiza en el banco de ensayos utilizando el Generador de trenes de pulsos de DP de referencia en HF, para 4 defectos tipo diferentes superpuestos en presencia de ruido eléctrico. Los nueve defectos tipo diferentes se eligen de los ficheros de la base de datos del generador confeccionada con las probetas descritas en la sección 3 y trenes de DP medidos en campo. 
La prueba consiste en reproducir nueve casos con mezcla de trenes de pulsos procedentes de cuatro defectos diferentes en cada caso. Los cuatro defectos incluidos en cada caso deben ser elegidos de forma que se utilice cada defecto cuatro veces en los nueve casos a generar. Las amplitudes de cada defecto perteneciente a un mismo caso son diferentes, con la condición de que en el valor seleccionado para la amplitud de la DP de mayor amplitud no supere 50 veces la amplitud de la DP de menor amplitud. A título de ejemplo se muestra el cuadro 2. Cada uno de los casos incluye la superposición de ruido eléctrico sinusoidal modulado, primero a nivel alto de amplitud iguala al 1,2 veces la amplitud de los pulsos del tren de DP de mayor amplitud y luego a nivel bajo, igual al $25 \%$ de amplitud de las DP de mayor amplitud. Cada uno de los trenes de pulsos de DP se genera con formas de onda pulsante de diferente contenido frecuencial y duración para simular las condiciones reales.

Cuadro 2. Ejemplo de superposición de defectos tipo.

\begin{tabular}{|c|c|c|c|c|c|c|c|c|c|}
\hline $\begin{array}{c}\text { Defecto } \\
\text { Tipo }\end{array}$ & $\# 1$ & $\# 2$ & $\# 3$ & $\# 4$ & $\# 5$ & $\# 6$ & $\# 7$ & $\# 8$ & $\# 9$ \\
\hline Caso 1 & $2 \%$ & & & & & & $100 \%$ & $50 \%$ & $25 \%$ \\
\hline Caso 2 & $25 \%$ & $2 \%$ & & & & & & $100 \%$ & $50 \%$ \\
\hline Caso 3 & $50 \%$ & $25 \%$ & $2 \%$ & & & & & & $100 \%$ \\
\hline Caso 4 & $100 \%$ & $50 \%$ & $25 \%$ & $2 \%$ & & & & & \\
\hline Caso 5 & $100 \%$ & $50 \%$ & $25 \%$ & $2 \%$ & & & & \\
\hline Caso 6 & & $100 \%$ & $50 \%$ & $25 \%$ & $2 \%$ & & & \\
\hline Caso 7 & & & $100 \%$ & $50 \%$ & $25 \%$ & $2 \%$ & & \\
\hline Caso 8 & & & & & $100 \%$ & $50 \%$ & $25 \%$ & $2 \%$ & \\
\hline Caso 9 & & & & & & $100 \%$ & $50 \%$ & $25 \%$ & $2 \%$ \\
\hline
\end{tabular}

\section{Eficacia para localizar la posición de los defectos}

La determinación de la eficacia para localizar la posición de los defectos se evalúa en dos ensayos similares a los ensayos de determinación de la eficacia para identificar automáticamente el tipo de defecto realizados en alta tensión, pero con al menos un par de sensores del mismo tipo a fin de que el sistema de medida pueda determinar el tiempo de vuelo de los pulsos de DP. El objetivo de la prueba es verificar que el defecto ha sido localizado correctamente en el elemento afectado (GIL o Cable) y que el error cometido en la determinación de la distancia entre los sensores y el defecto efectuada por el sistema a evaluar.

\section{Conclusiones}

Los sistemas de medidas y monitorización de descargas parciales convencionales y los no convencionales que utilizan sensores HF, VHF y UHF utilizados para el diagnóstico del estado del aislamiento pueden ser caracterizados funcionalmente mediante las pruebas definidas en este artículo como resultado del proyecto europeo FutureEnergy de EURAMET de apoyo a la normalización. Las pruebas caracterizan los sensores, el medidor y las herramientas de procesamiento de señal y diagnóstico. Las medidas en pC que son esenciales en los ensayos de aceptación de laboratorio y de recepción en campo que utilizan métodos de medida convencionales son sustituidos por verificaciones de sensibilidad de DP y evaluación de la eficacia para identificar patrones de DP resueltos en fase asociados a defectos representativos en GIS, cables y aislamientos en aire. Los trabajos realizados que se presentan en este artículo pretenden servir de apoyo a los grupos de normalización a fin de cubrir las lagunas normativas existentes en la actualidad. 


\section{Agradecimientos}

This project 19ENG02 FutureEnergy has received funding from the EMPIR programme co-financed by the Participating States and from the European Union's Horizon 2020 research and innovation programme.

\section{Referencias}

[1] IEC 60060-3 "High-voltage test techniques - Part 3: Definitions and requirements for on-site testing"

[2] IEEE 400.3 Guide for Partial Discharge Testing of Shielded Power Cable Systems in a Field Environment

[3] IEC 62271-203 High-Voltage Switchgear and controlgear-Part 203: Gas-insulated metal-enclosed switchgear for rated voltages above $52 \mathrm{kV}$ "

[4] UNE 211006 "Ensayos previos a la puesta en servicio de sistemas de cables eléctricos de alta tensión en corriente alterna"

[5] IEC 60270 High Voltage test techniques. Partial Discharge Measurements

[6] IEC TS 62478 High voltage test techniques - Measurement of partial discharges by electromagnetic and acoustic methods.

[7] "Effects of transient on High Voltage Cable Insulation", Dissertation doctoral Thesis, Jiayang Wu, 28th February 2020.

[8] "Application of HFCT and UHF Sensors in On-Line Partial Discharge Measurements for Insulation Diagnosis of High Voltage Equipment, Fernando Álvarez, Fernando Garnacho, Javier Ortego and Miguel Ángel SánchezUrán; Sensors 2015, 15, 7360-7387; doi:10.3390/s150407360

[9] "Development, testing and aging of reference insulation defects for the improvement in partial discharges diagnosis". Eduardo Arcones, Fernando Álvarez, Javier Ortego, Abderrahim Khamlichi, Álvaro Camuñas, Alejandro Rosete, Fernando Garnacho, Pablo Gómez.

[10] FutureEnergy "Metrology for future energy transmission". Start date: 01 June 2020 Duration: 36 months EMPIR Project EURAMET 